\title{
LECTURES ON WONDERFUL VARIETIES
}

\author{
GUIDO PEZZINI
}

\begin{abstract}
These notes are an introduction to wonderful varieties. We discuss some general results on their geometry, their role in the theory of spherical varieties, several aspects of the combinatorics arising from these varieties, and some examples.
\end{abstract}

\section{INTRODUCTION}

These notes are based on a series of lectures given by Michel Brion and Jacopo Gandini on wonderful varieties, in occasion of the "Workshop on Spherical Varieties", held from October 31 to November 4, 2016 at the Tsinghua Sanya International Mathematics Forum.

Wonderful varieties first appeared in the work of De Concini and Procesi, as compactifications (with remarkable properties) of symmetric spaces $G / G^{\theta}$, where $G$ is a semisimple linear algebraic group and $\theta: G \rightarrow G$ is an involution (see [DP83]). One of their motivations was to attack problems in classical enumerative geometry. These varieties include several ones studied in the theory of reductive groups, such as the variety of complete conics, or the space of complete collineations. Among the most important cases we may also mention the wonderful compactifications of adjoint semisimple groups, which have many relevant applications.

Later, Luna considered more general varieties, defining them wonderful if they have the same main properties of the De Concini-Procesi compactifications (see Definition 2.5). Such varieties have been then better understood, and have a central role, in the theory of spherical varieties. Knop proved in [Kn96] that, in a precise sense, there are "enough" wonderful varieties among the spherical ones (see Theorem 4.3 below). This enabled Luna to initiate in [Lu01] a classification program for spherical varieties, built around studying the wonderful case. This program has been completed recently, see [BP16] and references therein.

\section{ACKNOWLEDGEMENTS}

Many thanks are due to Baohua Fu and Michel Brion for organizing the workshop at the Tsinghua Sanya International Mathematics Forum. I also thank Michel Brion, Baohua Fu and Jacopo Gandini for useful exchanges on the topics of the lectures, and a referee for useful remarks and suggestions.

\section{Definitions AND EXAMPLES}

Throughout the paper, the ground field will be the field of complex numbers $\mathbb{C}$. We fix a connected reductive group $G$, and, unless otherwise stated, we fix a Borel subgroup $B \subseteq G$, a maximal torus $T \subseteq B$, we denote by $S$ the corresponding set of

Date: April 27, 2017. 
simple roots, and $W$ the Weyl group. Given an algebraic group $H$, we denote by $\mathcal{X}(H)$ its group of characters, by $H^{r}$ its radical, by $H^{u}$ its unipotent radical, and by $Z(H)$ its center.

Wonderful varieties are $G$-varieties where the orbit structure is particularly nice. Their structure is similar to the following basic example.

Example 2.1. Let $n \geq 1$ and $G=\left(\mathbb{C}^{*}\right)^{n}$, which acts on $\mathbb{C}^{n}$ by multiplication

$$
\left(t_{1}, \ldots, t_{n}\right) \cdot\left(x_{1}, \ldots, x_{n}\right)=\left(t_{1} x_{1}, \ldots, t_{n} x_{n}\right) .
$$

The $G$-stable prime divisors, i.e. the coordinate hyperplanes, are smooth and intersect transversally. The intersection of any subset of such divisors is non-empty and is an orbit closure, and all orbit closures are obtained in this way.

It is easy to give examples where the orbit structure does not fulfill these properties.

Example 2.2. The group $\operatorname{SL}(n)$ acting linearly on $\mathbb{C}^{n}$ (with $n \geq 2$ ) has two orbits, namely $\{0\}$ and its complement. The orbit $\{0\}$, which is closed, is not the intersection of $G$-stable prime divisors.

Our goal is to give a general definition for complete $G$-varieties, where the orbit structure resembles the one in Example 2.1. We start with the following.

Definition 2.3. Let $X$ be a smooth variety, and $D_{1}, \ldots, D_{r} \subset X$ prime divisors. The divisor $D=D_{1}+\ldots+D_{r}$ has strict normal crossings if, for all $p \in X$, any $D_{i}$ containing $p$ has a local equation in $\mathcal{O}_{X, p}$, and the set of these local equations can be completed to a local system of parameters of $\mathcal{O}_{X, p}$.

If a divisor $D=D_{1}+\ldots+D_{r}$ as above has strict normal crossings, then the components $D_{1}, \ldots, D_{r}$ are smooth. They induce a stratification of $X$ into locally closed smooth subvarieties, indexed by subsets $I \subseteq\{1, \ldots, r\}$, namely the varieties

$$
X_{I}=\left(\bigcap_{i \in I} D_{i}\right) \backslash\left(\bigcup_{j \notin I} D_{j}\right) .
$$

Notice that we do not require yet that the intersection $D_{1} \cap \ldots \cap D_{r}$ is non-empty.

Example 2.4. Let $n \geq 2$ and $X=\mathbb{P}\left(\mathbb{C}^{n} \oplus \mathbb{C}\right)$ with its natural action of $\operatorname{SL}(n)$, and define $Y=\mathrm{Bl}_{p}(X)$ where $p=[0, \ldots, 0,1]$. Then the action of $\operatorname{SL}(n)$ lifts to an action on $Y$, and $Y$ has exactly two $\mathrm{SL}(n)$-stable prime divisors. Their sum has strict normal crossings, but their intersection is empty.

We come to our main definition.

Definition 2.5 (Luna). Let $X$ be an irreducible $G$-variety. Then $X$ is wonderful (of rank $r$ ) if it is smooth, complete, it has exactly $r$ prime divisors $D_{1}, \ldots, D_{r}$ that are $G$-stable, and in addition they have strict normal crossings, non-empty intersection, and the stratum $X_{I}$ is a $G$-orbit for all $I \subseteq\{1, \ldots, r\}$.

Remark 2.6. Let $X$ be a wonderful $G$-variety of rank $r$.

(1) The variety $X$ has $2^{r} G$-orbits; among them, exactly one is open, namely $X_{\varnothing}$, and exactly one is closed, namely $X_{\{1, \ldots, r\}}$.

(2) Since $X$ is in particular normal with only one closed $G$-orbit, it follows by [Su74] that $X$ is projective, and a standard argument yields that the radical of $G$ acts trivially on $X$. 
Wonderful varieties play a crucial role in the theory of spherical varieties, let us recall the definition.

Definition 2.7. An irreducible normal $G$-variety $X$ is spherical if a Borel subgroup of $G$ has an open orbit on $X$.

A spherical variety has in particular an open $G$-orbit; the stabilizer $H$ of a point on this $G$-orbit (so that the latter is the homogeneous space $G / H$ ) is called a spherical subgroup of $G$.

The first observation we make to relate the two definitions above is the following.

Theorem 2.8 ([Lu96]). Any wonderful variety is spherical.

We point out that all requirements of Definition 2.5 are necessary to have sphericity, as seen in the next example.

Example 2.9 ([Lu96]). Consider $X=\mathbb{P}\left(\mathbb{C}^{2}\right) \times \mathbb{P}\left(\operatorname{Sym}^{2}\left(\mathbb{C}^{2}\right)\right)$ under the action of $G=\operatorname{SL}(2)$. Then $X$ is smooth and projective, it has exactly 4 orbits and two smooth $G$-stable prime divisors which intersect in a single $G$-orbit. More precisely, one $G$-stable prime divisor is $\mathbb{P}\left(\mathbb{C}^{2}\right) \times Y$, where $Y$ is the unique $G$-stable prime divisor of $\mathbb{P}\left(\operatorname{Sym}^{2}\left(\mathbb{C}^{2}\right)\right)$. We recall that any point of $\mathbb{P}\left(\operatorname{Sym}^{2}\left(\mathbb{C}^{2}\right)\right)$ can be written as a product $[v \cdot w]$ for some $v, w \in \mathbb{C}^{2} \backslash\{0\}$, and that $Y$ is the set of the points of the form $[v \cdot v]$ where $v \in \mathbb{C}^{2} \backslash\{0\}$. The other $G$-stable prime divisor of $X$ is the set of points $([u],[v \cdot w])$ with $u, v, w \in \mathbb{C}^{2} \backslash\{0\}$ such that $\mathbb{C} u=\mathbb{C} v$ or $\mathbb{C} u=\mathbb{C} w$. It is elementary to write local equations of these divisors, and conclude that they have the same tangent space in any point of their intersection, which is a single $G$-orbit. As a consequence, they don't have strict normal crossings, and $X$ is not spherical since it has dimension 3 . It is also possible to construct $\mathrm{SL}(2)$-varieties of dimension 3, where all $G$-stable prime divisors have strict normal crossings but empty intersection (see [MJ88]).

Let us give some examples of wonderful varieties.

Example 2.10. (1) An irreducible $G$-variety $X$ is wonderful of rank 0 if and only if it is homogeneous and complete. This is equivalent to being a partial flag variety $X=G / P$, where $P$ is a parabolic subgroup of $G$.

(2) An irreducible complete normal $G$-variety $X$ is wonderful of rank 1 if and only if it has two $G$-orbits, one open and one of codimension 1. Such varieties are classified, see [Ah83] and [Br88]. Let us give some examples.

(a) The product $X=\mathbb{P}^{1} \times \mathbb{P}^{1}$, with diagonal action of $G=\mathrm{SL}(2)$. The $G$-stable prime divisor is the diagonal.

(b) The variety $X=\mathbb{P}^{2}=\mathbb{P}\left(\operatorname{Sym}^{2}\left(\mathbb{C}^{2}\right)\right)$ under the action of $G=\operatorname{SL}(2)$.

(c) The variety $X=\mathbb{P}\left(M_{2}\right)$, where $M_{2}$ is the vector space of $(2 \times 2)$ matrices, under the action of $G=\mathrm{GL}(2) \times \mathrm{GL}(2)$ induced by left and right multiplication. The points corresponding to matrices of rank 1 form the $G$-stable prime divisor.

(3) Wonderful varieties of rank 2 have been classified in [Wa96]. A classical example is the variety of complete conics, which is the variety

$$
X=\left\{([A],[B]) \in \mathbb{P}\left(M_{3}\right) \times \mathbb{P}\left(M_{3}\right) \mid A B \in \mathbb{C} \cdot 1_{3 \times 3}\right\} .
$$

where we denote by $M_{n}$ the space of $(n \times n)$-matrices, and by $1_{n \times n}$ the unit matrix. The variety $X$ is the wonderful compactification of the homogeneous space $\mathrm{SL}(3) / \mathrm{SO}(3) Z(\mathrm{SL}(3))$, which is the space of smooth conics 
in $\mathbb{P}^{2}$. The two $G$-stable prime divisors of $X$ are:

$$
\begin{aligned}
& D_{1}=\{A \text { non-invertible }\} \\
& D_{2}=\{B \text { non-invertible }\}
\end{aligned}
$$

(4) Let us describe an example of rank 3 (see [Pe09, Section 3.5]). Let $G=$ $\operatorname{Sp}(2 a) \times \operatorname{Sp}(2 b)$ (with $a, b \geq 2$ ), and call $\Omega, \Omega^{\prime}$ the bilinear forms on $\mathbb{C}^{2 a}$ and $\mathbb{C}^{2 b}$ defining $\operatorname{Sp}(2 a)$ and $\operatorname{Sp}(2 b)$. For $k, n \in \mathbb{N}$, denote by $\operatorname{Gr}_{k}\left(\mathbb{C}^{n}\right)$ the Graßmannian of $k$-dimensional vector subspaces of $\mathbb{C}^{n}$. Consider the variety

$$
X=\left\{\begin{array}{l|l}
(E, F, p) & \begin{array}{l}
E \in \mathrm{Gr}_{2}\left(\mathbb{C}^{2 a}\right), \\
F \in \mathrm{Gr}_{2}\left(\mathbb{C}^{2 b}\right), \\
p \in \mathbb{P}(\operatorname{Hom}(E, F))
\end{array}
\end{array}\right\},
$$

where $G$ acts in a natural way. Under this action $X$ is wonderful of rank 3 , with $G$-stable prime divisors:

$$
\begin{aligned}
& D_{1}=\{\operatorname{rank} M=1\}, \\
& D_{2}=\left\{\left.\Omega\right|_{E_{1}}=0\right\}, \\
& D_{3}=\left\{\left.\Omega^{\prime}\right|_{E_{2}}=0\right\} .
\end{aligned}
$$

where we set $p=[M]$ for $M \in \operatorname{Hom}(E, F)$.

(5) An example of higher rank is given by the space of complete collineations (see [Se51]). It is a wonderful variety of rank $r$ for $G=\mathrm{GL}(r+1) \times \mathrm{GL}(r+1)$, where $r \geq 1$. We can describe it starting from the variety $Y=\mathbb{P}\left(M_{r+1}\right)$, which is a $G$-variety under left and right multiplication. Notice that $Y$ has $r+1$ orbits, whose closures $Z_{r+1} \supset Z_{r} \supset \ldots \supset Z_{1}$ are given by the condition that $Z_{i}$ is the set of points corresponding to matrices of rank at most $i$. While $Y$ is not a wonderful variety (if $r \geq 2$ ), we can blow up $Y$ successively along the (strict transform of) $Z_{1}, \ldots, Z_{r-1}$, and obtain a wonderful variety $X$. Its open $G$-orbit is isomorphic to $\operatorname{PGL}(r+1)$, hence $X$ is a wonderful compactification of this group.

We end this section with an easy observation on the geometry of wonderful varieties.

Proposition 2.11. Let $X$ be a wonderful variety of rank $r$ as in Definition 2.5, and $I \subseteq\{1, \ldots, r\}$. Then

$$
X^{I}=\overline{X_{I}}=\bigcap_{i \in I} D_{i}
$$

is a wonderful variety, of rank $r-|I|$.

\section{The LOCAL StRUCtURE THEOREM}

We discuss in this section the fundamental result of Brion, Luna, and Vust on the local structure of $G$-varieties, applied to the wonderful case.

Let $X$ be a wonderful $G$-variety of rank $r$. Its closed $G$-orbit $Y$ is a complete homogeneous space for $G$, so it is isomorphic to a quotient of the form $G / Q$, where $Q$ is the stabilizer of a point $y \in Y$, and it is a parabolic subgroup of $G$.

Denote by $P$ a parabolic subgroup of $G$ opposite to $Q$, i.e. such that $P \cap Q=L$ is a Levi subgroup of $P$ and $Q$. Denote by $P^{u}$ the unipotent radical of $P$. 
Theorem 3.1 ([BLV86]). Under the above assumptions, there exists a locally closed affine subvariety $Z \subseteq X$ containing y such that $Z$ is L-stable, and such that the map

$$
\begin{array}{cc}
P^{u} \times Z & \rightarrow X \\
(g, x) & \mapsto g x
\end{array}
$$

is an open $P$-equivariant immersion, where an element $p \in P$ acts on $P^{u} \times Z$ as $v l \cdot(u, z)=\left(v l u l^{-1}, l z\right)$, where $u, v \in P^{u}, l \in L, z \in Z$, and $p=v l$.

The above theorem holds in this form for varieties that are much more general than wonderful ones. However, since $X$ here is wonderful, the structure of the variety $Z$ is very particular, and motivates further the analogy with Example 2.1.

Proposition 3.2. A variety $Z$ as in Theorem 3.1 is isomorphic to the affine space $\mathbb{A}^{r}$, on which $L$ acts as $g \cdot\left(u_{1}, \ldots, u_{r}\right)=\left(\sigma_{1}(g) u_{1}, \ldots, \sigma_{r}(g) u_{r}\right)$ where $\sigma_{1}, \ldots, \sigma_{r}$ are linearly independent characters of $L$. Moreover, under the isomorphism $Z \cong \mathbb{A}^{r}$, the intersection $D_{i} \cap Z$ is identified with $\left\{u_{i}=0\right\}$ for all $i \in\{1, \ldots, r\}$.

Proof. Consider the point $y \in Z \cap Y$ as above. Recall that the orbit map $P^{u} \rightarrow Y$ sending $g$ to $g y$ is an open immersion by the Bruhat decomposition of $Y$. Then Theorem 3.1 implies $Z \cap Y=\{y\}$, and also that $Z$ is smooth of dimension $r$.

Since $X$ is wonderful, there exist local coordinates $t_{1}, \ldots, t_{r}$ around $y$ such that $t_{i}$ is a local equation of $D_{i}$ for all $i$. Theorem 3.1 implies the restriction $v_{i}=\left.t_{i}\right|_{Z}$ is a local equation of $D_{i} \cap Z$ in $Z$ in a neighborhood of the point $y$.

We may suppose that $v_{i} \in \mathbb{C}[Z]$. Then the classes $\overline{v_{1}}, \ldots, \overline{v_{r}}$ are a basis of $\mathfrak{m}_{y} / \mathfrak{m}_{y}^{2}$, where we denote by $\mathfrak{m}_{y}$ the maximal ideal in $\mathbb{C}[Z]$ of the point $y$.

The ideal $\mathfrak{m}_{y}$ is a rational $L$-module, and $\mathfrak{m}_{y}^{2}$ is a submodule. Since $D_{i} \cap Z$ is $L$-stable for all $i$, the class $\overline{v_{i}}$ is an $L$-eigenvector. Since $L$ is reductive, we can lift these classes to $L$-eigenvectors $u_{1}, \ldots, u_{r} \in \mathfrak{m}_{y}$. Notice that $(L, L)$ fixes $u_{1}, \ldots, u_{r}$, therefore it acts trivially on $Z$.

The $L$-eigenvectors $u_{1}, \ldots, u_{r} \in \mathfrak{m}_{y}$ have linearly independent weights, because otherwise a monomial $u_{1}^{n_{1}} \cdots u_{r}^{n_{r}}$ (with exponents not all zero) would be a nonconstant element of $\mathbb{C}(Z)^{L}$. But the latter is equal to $\mathbb{C}(X)^{P}$ by Theorem 3.1, and this field is $\mathbb{C}$ by Theorem 2.8. We also deduce that $L$ has an open orbit on $Z$.

At this point $\left[\right.$ Lu73, Corollaire 2, p. 98] implies that $Z=\operatorname{Spec} \mathbb{C}\left[u_{1}, \ldots, u_{r}\right] \cong \mathbb{A}^{r}$. Moreover, the subsets $\left\{u_{1}=0\right\}, \ldots,\left\{u_{r}=0\right\}$ of $Z$ are its unique $L$-stable prime divisors, hence they coincide with resp. $D_{1} \cap Z, \ldots, D_{r} \cap Z$.

From now on, we fix a Borel subgroup $B \subseteq G$ contained in $P$. We give some definitions for the wonderful variety $X$, taken from the theory of spherical varieties.

Definition 3.3. With the above notations, we define:

(1) the set $\Delta(X)$ of the $B$-stable, not $G$-stable prime divisors of $X$, called the colors of $X$,

(2) the set $\Sigma(X)=\left\{\sigma_{1}, \ldots, \sigma_{r}\right\}$, whose elements are called the spherical roots of $X$.

(3) the lattice $\Xi(X)$ of the $B$-eigenvalues of $B$-eigenvectors of $\mathbb{C}(X)$.

Remark 3.4. Thanks to Proposition 3.2, the spherical roots of $X$ are also the weights of $T$ acting on the normal space $T_{X, y} / T_{Y, y}$.

Example 3.5. It is not difficult to compute $\Sigma(X)$ for $X$ equal to the space of complete conics, using the definition we have given in Example 2.10: we have $\Sigma(X)=\left\{2 \alpha_{1}, 2 \alpha_{2}\right\}$, where $\alpha_{1}, \alpha_{2}$ are the simple roots of $\mathrm{SL}(3)$. 
Lemma 3.6. The set $\Sigma(X)$ is a basis of $\Xi(X)$.

Proof. Let $\mathbb{C}(X)^{(B)}$ be the multiplicative group of rational functions on $X$ that are $B$-eigenvectors. By Theorem 3.1 we have $\mathbb{C}(X)^{(B)}=\mathbb{C}(Z)^{(B \cap L)}$, and the lemma follows from Proposition 3.2.

Proposition 3.7. The open B-orbit of $X$ is equal to $P^{u} \cdot Z_{0}$, where $Z_{0}$ is the open $L$-orbit of $Z$. Moreover, we have

$$
X \backslash\left(P^{u} \cdot Z\right)=\bigcup_{D \in \Delta(X)} D .
$$

Proof. The first assertion is clear thanks to Theorem 3.1 and Proposition 3.2, let us show the second one.

Let $D \in \Delta(X)$. If $D$ intersects $P^{u} \cdot Z$ then the intersection is a $B$-stable prime divisor of $P^{u} \cdot Z \cong P^{u} \times Z$, so it is of the form $P^{u} \times E$ where $E$ is a $(B \cap L)$ stable prime divisor of $Z$. But then $E$ is one of coordinate hyperplanes of $Z$, and $D \cap\left(P^{u} \cdot Z\right)$ coincides with $D_{i} \cap\left(P^{u} \cdot Z\right)$ for some $i$. This yields $D=D_{i}$, contradicting the assumption that $D$ is not $G$-stable. So $D \subseteq X \backslash\left(P^{u} \cdot Z\right)$.

Conversely, the set $X \backslash\left(P^{u} \cdot Z\right)$ has pure codimension 1 , since $P^{u} \cdot Z \cong P^{u} \times Z$ is affine. Let $D$ be one of its irreducible components: it is $B$-stable because $P^{u} \cdot Z$ is $B$-stable, and $D$ is not $G$-stable because it cannot contain the unique closed $G$-orbit $Y$. Hence $D \in \Delta(X)$, and the proof is complete.

Proposition 3.8 ([Br89]). The Picard group of $X$ is freely generated by the classes of the colors, and the classes of the G-stable prime divisors are linearly independent.

Proof. Let $D$ be a divisor of $X$, and let $D_{0}$ be the linear combination with same coefficients only of the prime divisors of $D$ that are not colors. Since $X_{0}=X$ 入 $\bigcup_{D \in \Delta(X)} D$ is an affine space, the divisor $D_{0}$ is principal in $X_{0}$, i.e. $D_{0}=(f)$ for some $f \in \mathbb{C}\left(X_{0}\right)$. But then $D-(f)$, where the divisor $(f)$ is taken now in $X$, is a linear combination of colors.

In other words, up to linear equivalence we may assume $D_{0}=0$. If now $D$ is principal in $X$, with $D=(f)$ for some $f \in \mathbb{C}(X)$, then $\left.f\right|_{X_{0}}$ is a nowhere-vanishing regular function on $X_{0}$. It follows that $f$ is constant, and $D=0$.

This shows the first assertion of the proposition. To show the second, suppose that a linear combination $a_{1} D_{1}+\ldots+a_{r} D_{r}$ is principal, so it is equal to $(f)$ for some $f \in \mathbb{C}(X)$. Then $f$ restricts to a nowhere-vanishing regular function on the open $G$-orbit $G / H$ of $X$. Pull-back $f$ along the quotient $G \rightarrow G / H$. By [KKV89, Proposition 1.2], this pull-back is a character of $G$ up to a multiplicative constant: since the radical of $G$ acts trivially on $X$, we conclude that $f$ is constant and so the coefficients $a_{1}, \ldots, a_{r}$ are all 0.

Thanks to the above proposition, we can express for all $i$ the class $\left[D_{i}\right]$ of $D_{i}$ as a linear combination of the classes of the colors, enabling us to give the following.

Definition 3.9. We define the Cartan pairing of $X$ as the map

$$
c: \Delta(X) \times \Sigma(X) \rightarrow \mathbb{Z}
$$

such that

$$
\left[D_{i}\right]=\sum_{D \in \Delta(X)} c\left(D, \sigma_{i}\right)[D]
$$

in the Picard group of $X$, for all $i \in\{1, \ldots, r\}$. 
Often the Cartan pairing is extended linearly to a map

$$
c: \operatorname{span}_{\mathbb{Z}} \Delta(X) \times \operatorname{span}_{\mathbb{Z}} \Sigma(X) \rightarrow \mathbb{Z} \text {. }
$$

Remark 3.10. To compute the Cartan pairing in practice, we can use Propositions 3.2 and 3.7. They imply that a $G$-stable prime divisor $D_{i}$ of a wonderful variety $X$ has a local equation $f_{i} \in \mathbb{C}(X)$ around the point $y$, such that $f_{i}$ is a $B$-eigenvector of $B$-eigenvalue $-\sigma_{i}$. Moreover, thanks to the two propositions mentioned above, the order of $f_{i}$ along any other $G$-stable prime divisor is 0 , and any other zero or pole of $f_{i}$ is a color. It follows that the corresponding principal divisor is $D_{i}-\sum_{D \in \Delta(X)} c\left(D, \sigma_{i}\right) D$. We may rephrase this analysis as follows: the Cartan pairing $c\left(D, \sigma_{i}\right)$, for $D \in \Delta(X)$ is equal to the valuation of $f_{i}^{-1}$ along the prime divisor $D$.

Example 3.11. Assume $G$ is adjoint, and identify it with the $G \times G$-homogeneous (symmetric) space $X_{0}=G \times G / \operatorname{diag}(G)$. Then $X_{0}$ has a $G \times G$-equivariant wonderful completion $X$ thanks to the work of De Concini and Procesi. Let us denote by $B_{-}$the Borel subgroup of $G$ opposite to $B$ with respect to $T$; we fix the Borel subgroup $B \times B_{-}$of $G \times G$ and the maximal torus $T \times T$.

We have $\Xi(X)=\{(\chi,-\chi) \mid \chi \in \mathcal{X}(T)\}$. The spherical roots of $X$ are the elements $(\alpha,-\alpha)$, where $\alpha$ is any simple root of $G$. We can also choose the Borel subgroup $B \times B$ of $G \times G$ instead. With this choice, we consider the set of simple roots of $G \times G$ as the union of the sets of simple roots of the two factors $G$. For a simple root $\alpha$ of the first factor $G$, define $\alpha^{\prime}$ in such a way that $-w_{0}\left(\alpha^{\prime}\right)$ is the simple root of the second factor $G$ corresponding to $\alpha$, where $w_{0}$ is the longest element of the Weyl group of $G$. Then the spherical roots of $X$ are the set

$$
\Sigma(X)=\left\{\alpha+\alpha^{\prime} \mid \alpha \text { simple root of } G\right\}
$$

Let us get back to the choice of the Borel subgroup $B \times B_{-} \subseteq G \times G$. The colors of $X$ are the closures of the $B \times B_{-}$-stable prime divisors of $X_{0}$, and these are the irreducible components of $X_{0} \backslash B B_{-}$, since $B B_{-}$is the open $B \times B_{-}$-orbit of $X_{0}$.

Let us compute the Cartan pairing $c: \Delta(X) \times \Sigma(X) \rightarrow \mathbb{Z}$. Denote by $\widetilde{G}$ the universal cover of $G$, and by $\omega_{\alpha}$ the fundamental dominant weight corresponding to a simple root $\alpha$ of $G$. Let $V\left(\omega_{\alpha}\right)$ be the irreducible $\widetilde{G}$-module of highest weight $\omega_{\alpha}$, choose a highest weight vector $v_{\alpha} \in V\left(\omega_{\alpha}\right)$, and a lowest weight vector $\eta_{\alpha}$ in the dual module $V\left(\omega_{\alpha}\right)^{*}$. Recall that $\eta_{\alpha}$ has weight $-\omega_{\alpha}$.

With these assumptions the matrix coefficient $F_{\alpha}: g \mapsto\left\langle\eta_{\alpha}, g^{-1} v_{\alpha}\right\rangle$ is a global equation in $\mathbb{C}[\widetilde{G}]$ of a divisor $\widetilde{D}_{\alpha}$ of $\widetilde{G}$. We have that $\widetilde{D}_{\alpha}$ is the pull-back on $\widetilde{G}$ of a $B \times B_{-}$-stable prime divisor $D_{\alpha, 0}$ of $X_{0}$, so the closure $D_{\alpha}=\overline{D_{\alpha, 0}}$ in $X$ is a color of $X$. All colors of $X$ are obtained in this way.

Finally, the function $F_{\alpha}$ is $\left(B \times B_{-}\right)$-semiinvariant, of weight $\left(\omega_{\alpha},-\omega_{\alpha}\right)$, hence the product

$$
f_{\alpha}=\prod_{\beta \text { simple root }} F_{\beta}^{\left\langle\alpha, \beta^{\vee}\right\rangle}
$$

is a $\left(B \times B_{-}\right)$-semiinvariant rational function on $X$ with $\left(B \times B_{-}\right)$-weight equal to $(\alpha,-\alpha)$. Thanks to Remark 3.10, we conclude that $c\left(D_{\alpha},(\alpha,-\alpha)\right)$ is equal to the order of $f_{\alpha}$ along $D_{\alpha}$, i.e. the Cartan pairing $c$ of $X$ is given by the Cartan matrix of $G$. 


\section{The LOGARITHMiC TANGENT BUNDLE}

We begin this section recalling some basic facts on a $G$-variety $X$, only assuming it is smooth and irreducible. For more details, we refer to [Br07, Section 2].

For a point $x \in X$, consider the orbit map $G \rightarrow X$ sending $g$ to $g x$. Its differential in the neutral element $e \in G$ is a linear map

$$
\theta_{x}: \mathfrak{g} \rightarrow T_{X, x}
$$

where $\mathfrak{g}$ is the Lie algebra of $G$ and $T_{X, x}$ is the tangent space of $X$ in $x$. Notice that $\theta_{x}$ is surjective if and only if the orbit $G \cdot x$ is open in $X$.

This picture can be globalized: denote by $\mathcal{T}_{X}$ the sheaf of sections of the tangent bundle of $X$, and denote by $\mathfrak{g}$ the constant sheaf on $X$ associated to $\mathfrak{g}$. Then the $G$-action induces a morphisms of sheaves on $X$

$$
\theta: \mathcal{O}_{X} \otimes \underline{\mathfrak{g}} \rightarrow \mathcal{T}_{X} .
$$

Notice that $X$ is $G$-homogeneous if and only if this morphism of sheaves is surjective.

Let now $D=D_{1}+\ldots+D_{r}$ be a divisor on $X$ with strict normal crossings as in Definition 2.3. Considering $\mathcal{T}_{X}$ as the sheaf of derivations $\operatorname{Der}\left(\mathcal{O}_{X}\right)$, we can consider the subsheaf of derivations stabilizing the ideal sheaf $\mathcal{O}_{X}(-D)$ of $D$, and we denote it by $\mathcal{T}_{X}(-D)$. It is locally free, and it is called the logarithmic tangent sheaf of the pair $(X, D)$.

Remark 4.1. Let $n=\operatorname{dim}(X)$, so $n \geq r$. Fix $x \in X$, and denote by $I$ the subset of $\{1, \ldots, r\}$ such that $x \in D_{i}$ if and only if $i \in I$. Denote by $t_{1}, \ldots, t_{n}$ local coordinated around $x$, such that $t_{i}=0$ is a local equation of $D_{i}$ for all $i \in I$. Then $\mathcal{T}_{X}(-D)$ is generated, locally around $x$, by $t_{i} \frac{\partial}{\partial t_{i}}$ for $i \in I$, and by $\frac{\partial}{\partial t_{j}}$ for $j \in\{1, \ldots, n\} \backslash I$.

If $G$ acts on $X$ preserving $D$, then $\theta$ maps $\mathcal{O}_{X} \otimes \underline{\mathfrak{g}}$ to $\mathcal{T}_{X}(-D)$, and we have the following.

Proposition 4.2. If $X$ is wonderful then $\theta: \mathcal{O}_{X} \otimes \underline{\mathfrak{g}} \rightarrow \mathcal{T}_{X}(-D)$ is surjective. Moreover, the kernel $\operatorname{ker} \theta_{x}$ (contained in the stabilizer $\mathfrak{g}_{x}$ ) is the kernel of the $\mathfrak{g}_{x}$-action on the normal space $T_{X, x} / T_{G x, x}$.

Proof (sketch). The proof is reduced, thanks to Theorem 3.1 and Proposition 3.2, to the elementary case of Example 2.1.

The above proposition implies that, for all points $x$ of an $n$-dimensional wonderful variety $X$, the kernel $\operatorname{ker} \theta_{x}$ is a Lie subalgebra of $\mathfrak{g}$ of codimension $n$. This induces the Demazure morphism

$$
\begin{aligned}
f: \quad X & \rightarrow \operatorname{Gr}^{n}(\mathfrak{g}) \\
x & \mapsto \operatorname{ker} \theta_{x}
\end{aligned}
$$

where $\operatorname{Gr}^{n}(\mathfrak{g})$ is the Graßmannian of $n$-codimensional vector subspaces of $\mathfrak{g}$.

We end this section summarizing in the following theorem several important results on wonderful varieties.

Theorem 4.3. Let $X$ be a wonderful variety, with open $G$-orbit $G / H$.

(1) The quotient $N_{G}(H) / H$ is finite.

(2) If $H=N_{G}(H)$ then $f: X \rightarrow \mathrm{Gr}^{n}(\mathfrak{g})$ is an immersion. In particular $N_{G}(H)$ is the stabilizer of the Lie algebra $\mathfrak{h}$ of $H$ under the adjoint action of $G$ on $\mathfrak{g}$. 
(3) For any spherical subgroup $K \subseteq G$ such that $K=N_{G}(K)$ the homogeneous space $G / K$ admits a wonderful completion.

Proof (references). The first assertion follows from [BrPa87, Corollaire 5.3]. The second is proved in [Lo09b]. The third assertion is a fundamental theorem of Knop, see $[\mathrm{Kn} 96]$.

\section{SPHERICAL ROOTS AND COLORS}

The finite sets $\Delta(X)$ and $\Sigma(X)$, which we attach to any wonderful $G$-variety $X$, have a quite rich combinatorial structure. It generalizes the combinatorial part of the theory of symmetric spaces.

The first main result we mention is Theorem 5.1 below. Before stating it, we recall that the spherical roots can be defined also for a general spherical variety. Correspondingly, Theorem 5.1 can be stated also for a general spherical variety; it is due to Brion in characteristic 0 (see [Br90]), and to Knop in odd characteristic (see $[$ Kn14]).

Let $X$ be a wonderful $G$-variety. We equip $\Xi(X) \otimes_{\mathbb{Z}} \mathbb{Q}$ with an inner product by restricting a $W$-invariant inner product on $\mathcal{X}(T) \otimes_{\mathbb{Z}} \mathbb{Q}$.

Theorem 5.1 ([Br90, Kn14]). Let $X$ be a wonderful variety. The set $\Sigma(X)$ is the set of simple roots of a root system.

We denote by $W(X)$ the Weyl group of the root system generated by $\Sigma(X)$. It is called the little Weyl group of $X$, and generalizes the little Weyl group classically defined for symmetric spaces.

Also the set of colors $\Delta(X)$, equipped with the Cartan pairing $c: \Delta(X) \times \Sigma(X) \rightarrow$ $\mathbb{Z}$, plays a crucial role in this picture, and in some sense generalizes the set of simple coroots of $\Sigma(X)$. This analogy is suggested first of all by Example 3.11, and also by the rigid combinatorial properties of $\Delta(X)$ we will see in this section, but it must not be taken literally, since $\Delta(X)$ is not in general the set of simple coroots of $\Sigma(X)$.

Let us give in the next proposition a first view of the interplay between the combinatorics of $\Delta(X)$ and $\Sigma(X)$, and the geometry of $X$. In the proof we will use some results from [Kn91] concerning $G$-equivariant morphisms between wonderful varieties. They also have a very effective combinatorial counterpart, and will be recalled in details in Section 7.

Proposition 5.2. Let $X$ a wonderful $G$-variety with open orbit $G / H$, and denote by $P$ the parabolic subgroup as in Section 3. Then the following hold.

(1) $\operatorname{dim}(X)=\operatorname{dim} P^{u}+|\Sigma(X)|$.

(2) $\operatorname{rk}(\mathcal{X}(H))=|\Delta(X)|-|\Sigma(X)|$.

(3) The subgroup $H$ is reductive if and only if there exists a linear combination $\sigma$ of spherical roots with non-negative coefficients, such that $\langle D, \sigma\rangle>0$ for all $D \in \Delta(X)$.

(4) The subgroup $H$ is very reductive, i.e. it is not contained in any proper parabolic subgroup of $G$, if and only if $|\Delta(X)|=|\Sigma(X)|$.

Proof. Part (1) follows from Theorem 3.1 and Proposition 3.2.

Let us show part (2). Recall that $\Delta(X)$ is a basis of $\operatorname{Pic}(X)$. Up to the natural identification of $\Sigma(X)$ with the set of $G$-stable prime divisors of $X$, the desired equality follows putting together the following: 
(1) the short exact sequence

$$
0 \rightarrow \operatorname{span}_{\mathbb{Z}} \Sigma(X) \rightarrow \operatorname{Pic}(X) \rightarrow \operatorname{Pic}(G / H) \rightarrow 0,
$$

(2) the isomorphism

$$
\operatorname{Pic}(G / H) \cong \mathcal{X}(H)
$$

which holds thanks to [KKV89, Proposition 3.2] and the fact that we may assume $G$ semisimple and simply connected.

Part (3) is shown in [Kn91], let us show part (4). Let us denote by $C$ the convex cone generated in the vector space $N=\operatorname{Hom}_{\mathbb{Z}}(\Xi(X), \mathbb{Q})$ by the elements $c(D,-)$ for $D$ varying in $\Delta(X)$, and by $V$ the convex cone generated by the elements $-\sigma_{1}^{*}, \ldots,-\sigma_{r}^{*}$, where $\sigma_{1}^{*}, \ldots, \sigma_{r}^{*}$ is the dual basis of $\Sigma(X)$.

We recall that, by [Kn91], the vector space $N$ is generated, as a convex cone, by $C$ and $V$. Assume that $|\Delta(X)|=|\Sigma(X)|$. Then, by Proposition 3.8, the elements $c(D,-)$ for $D$ varying in $\Delta(X)$ are linearly independent. The consequences are that $C$ is strictly convex, and that the map $\operatorname{Pic}(X) \rightarrow N$ induced by the Cartan pairing is injective.

We claim that $C \cap V=\{0\}$. Otherwise, there exist non-negative coefficients $n_{1}, \ldots, n_{r}$ and $m_{D}$ for $D \in \Delta(X)$ such that

$$
n_{1} D_{1}+\ldots+n_{r} D_{r}+\sum_{D \in \Delta(X)} m_{D} D
$$

is a principal divisor, and not all coefficients are zero. But, being $X$ complete, any effective principal divisor must be zero: contradiction. This proves the claim, which in turn implies that the linear combination of part (3) exists.

Therefore $H$ is reductive. If it is contained in a proper parabolic subgroup $Q$ of $G$, then $H$ is contained in a Levi subgroup of $Q$, so there exists a non-trivial torus $T^{\prime} \subseteq Q$ centralizing $H$. If $T^{\prime}$ is not contained in $H$ then the first assertion of Theorem 4.3 is contradicted, so $T^{\prime} \subseteq H$. But then $\mathcal{X}(H)$ has positive rank, contradicting part (2). We conclude that $H$ is very reductive.

Vice versa, assume $H$ is not contained in any proper parabolic subgroup of $G$. Then it is well known that $H$ is reductive, hence by part $(3)$ the elements $c(D,-)$ for $D$ varying in $\Delta(X)$ generate a strictly convex cone inside $\operatorname{Hom}_{\mathbb{Z}}(\Xi(X), \mathbb{Q})$.

Suppose, for sake of contradiction, that $|\Delta(X)|>|\Sigma(X)|$. This means in particular that $|\Delta(X)|$ is greater than the dimension of $N$. Recall that this vector space is generated, as a convex cone, by the sets $C$ and $V$. Then one can show, with an elementary argument on convex polytopes, that there is at least one $E \in \Delta(X)$ such that $N$ is generated, as a convex cone, by $V$ together with the elements $c(D,-)$ for $D$ varying in $\Delta(X) \backslash\{E\}$.

By [Kn91, Theorem 5.4], there exists a $G$-equivariant morphism $X \rightarrow G / Q$, where $Q$ is a parabolic subgroup of $G$, such that $E$ is the inverse image of a color of $G / Q$. This implies that $Q$ is a proper subgroup of $G$, and it contains $H$ up to conjugation. This contradicts our assumptions, and the proof is complete.

The colors of a wonderful $G$-variety are also related to the simple roots of $G$, as in the following.

Definition 5.3. A color $D$ of a wonderful $G$-variety $X$ is moved by a simple root $\alpha$ of $G$ if $D$ is not stable under the minimal parabolic subgroup $P_{\alpha}$ strictly containing $B$ and associated to $\alpha$. We denote by $S^{p}(X)$ the set of simple roots moving no color. 
By Proposition 3.7, the parabolic subgroup $P$ of Section 3 is the common stabilizer of all colors, so it is the parabolic subgroup of $G$ containing $B$ and corresponding to $S^{p}(X)$.

As we will remark later, there are compatibility properties satisfied by $S^{p}(X)$ and $\Sigma(X)$. Let us see one following directly from the local structure of $X$.

Lemma 5.4. If $\alpha \in S^{p}(X)$, then $\left\langle\alpha^{\vee}, \sigma\right\rangle=0$ for all $\sigma \in \Sigma(X)$.

Proof. Let $L$ be the Levi subgroup of $P$ containing $T$. From Proposition 3.2 we have that the restriction of $\sigma$ to the maximal torus $T \cap(L, L)$ of $(L, L)$ is zero. The lemma follows.

Example 5.5. If $G=\mathrm{SL}(2)$, then there exist exactly 4 wonderful $G$-varieties up to isomorphism. They are the following.

- A single point: it has no color and no spherical root.

- The variety $X=\mathbb{P}^{1}=G / B$ : it has one color but no spherical root (the Cartan pairing is the empty map).

- The variety $X=\mathbb{P}^{1} \times \mathbb{P}^{1}$, where $\mathrm{SL}(2)$ acts diagonally. Denote by $\alpha$ the simple root of SL(2); then $X$ has two colors $D_{\alpha}^{+}$and $D_{\alpha}^{-}$, one spherical root $\alpha$, and $c\left(D_{\alpha}^{ \pm}, \alpha\right)=1$

- The variety $X=\mathbb{P}^{2}=\mathbb{P}\left(\operatorname{Sym}^{2}\left(\mathbb{C}^{2}\right)\right)$. It has one color $D_{\alpha}$ and one spherical root $2 \alpha$, with $c\left(D_{\alpha}, 2 \alpha\right)=2$.

All colors of these varieties are moved by the simple root $\alpha$ of $G$, since in this case $P_{\alpha}=G$.

In [Lu97], Luna showed in general that a simple root cannot move arbitrarily many colors, using a reduction to the case of the above example. He also showed that the values of the Cartan pairing are strongly influenced by which simple roots move which colors, and in [Lu01] that only in specific situations the same color can be moved by two different simple roots.

We summarize these results in the following.

Proposition 5.6 ([Lu97, Lu01]). Let $X$ be a wonderful $G$-variety.

(1) A simple root $\alpha$ moves at most two colors. It moves two colors, denoted by ${ }^{1}$ $D_{\alpha}^{+}, D_{\alpha}^{-}$, if and only if $\alpha \in \Sigma(X)$, in which case $c\left(D_{\alpha}^{+},-\right)+c\left(D_{\alpha}^{-},-\right)=$ $\alpha^{\vee} \mid \Xi(X)$.

(2) If a color $D$ is moved by $\alpha \in S \cap \Sigma(X)$ and $c(D, \sigma)>0$ for some $\sigma \in \Sigma(X)$, then $\sigma$ is a simple root, it moves $D$, and $c(D, \sigma)=1$.

(3) Two different simple roots $\alpha$ and $\beta$ move the same color $D$ if and only if one of the two following mutually exclusive situations occur.

(a) Both $\alpha$ and $\beta$ are spherical roots, and they move the same color.

(b) The simple roots $\alpha$ and $\beta$ are orthogonal, and $\alpha+\beta$ or $\frac{1}{2}(\alpha+\beta)$ is in $\Sigma(X)$.

(4) If $2 \alpha \in \Sigma(X)$ with $\alpha$ a simple root, then $\alpha$ moves only one color $D$, and $c(D,-)=\frac{1}{2} \alpha^{\vee} \mid \Xi(X)$.

(5) If a simple root $\alpha$ satisfies $\alpha, 2 \alpha \notin \Sigma(X)$ and moves one color $D$, then $c(D,-)=\left.\alpha^{\vee}\right|_{\Xi(X)}$.

\footnotetext{
${ }^{1}$ There is in general no preferred way to decide which color is denoted by $D_{\alpha}^{+}$and which by $D_{\alpha}^{-}$. However, whenever possible, it is common to choose them in such a way that $c\left(D_{\alpha}^{+},-\right) \geq c\left(D_{\alpha}^{-},-\right)$.
} 
Example 5.7. Let us illustrate in an example statements (2) and (3a) of Proposition 5.6. Let $G=\mathrm{SL}(3)$, and let $H_{1}$ be the subgroup of matrices of the form

$$
\left(\begin{array}{lll}
1 & x & * \\
0 & 1 & x \\
0 & 0 & 1
\end{array}\right)
$$

for $x \in \mathbb{C}$. Define $H=N_{G}\left(H_{1}\right)$. Then $G / H$ has a wonderful completion, with spherical roots $\alpha_{1}, \alpha_{2}$ (see [Av15] for more details of such subgroups). It has three colors: one, denoted by $D_{\alpha_{1}}^{+}=D_{\alpha_{2}}^{+}$, is moved by $\alpha_{1}$ and $\alpha_{2}$, the others $D_{\alpha_{1}}^{-}$and $D_{\alpha_{2}}^{-}$are moved resp. by $\alpha_{1}$ and $\alpha_{2}$. The Cartan pairing is

\begin{tabular}{c|cc} 
& $\alpha_{1}$ & $\alpha_{2}$ \\
\hline$D_{\alpha_{1}}^{+}$ & 1 & 1 \\
$D_{\alpha_{1}}^{-}$ & 1 & -2 \\
$D_{\alpha_{2}}^{-}$ & -2 & 1
\end{tabular}

A similar example can be built in any simple group $G$, setting $H=N_{G}\left(H_{1}\right)$ and defining $H_{1}$ to be a subgroup of $B^{u}$ containing the commutator $\left(B^{u}, B^{u}\right)$ and such that the Lie algebra of $H_{1}$ contains a one-dimensional subspace "diagonal" in the sum

$$
\bigoplus_{\alpha \text { simple root }} \mathfrak{g}_{\alpha}
$$

where $\mathfrak{g}_{\alpha}$ is the root space of the Lie algebra $\mathfrak{g}$ of $G$ corresponding to the root $\alpha$ (see also Example 8.3(3)). The spherical roots of the corresponding wonderful variety are the simple roots of $G$. Each simple root $\alpha$ moves two colors $D_{\alpha}^{+}, D_{\alpha}^{-}$, and we have $D_{\alpha}^{+}=D_{\beta}^{+}$for all simple roots $\alpha, \beta$. The value of $c\left(D_{\alpha}^{+}, \alpha\right)$ is 1 for any simple root $\alpha$, whereas for all simple roots $\alpha, \beta$ we have $c\left(D_{\alpha}^{-}, \beta\right)=\left\langle\beta, \alpha^{\vee}\right\rangle-1$, according to statement (1) of Proposition 5.6. For example, if $G$ has type $\mathrm{B}_{2}$ the resulting Cartan matrix is

\begin{tabular}{c|cc} 
& $\alpha_{1}$ & $\alpha_{2}$ \\
\hline$D_{\alpha_{1}}^{+}$ & 1 & 1 \\
$D_{\alpha_{1}}^{-}$ & 1 & -2 \\
$D_{\alpha_{2}}^{-}$ & -3 & 1
\end{tabular}

Example 5.8. An example of the situation of statement (3b) of Proposition 5.6 is given by the varieties $X$ of Example 3.11, under the action of $G \times G$ for $G$ an adjoint group. Recall that we choose $B \times B_{-}$as a Borel subgroup of $G \times G$. As described in Example 3.11, the variety $X$ has a color $D_{\alpha}$ for each simple root $\alpha$ of $G$, and the Cartan pairing between $D_{\alpha}$ and the spherical root $(\beta,-\beta)$ (for $\beta$ simple root of $G$ ) is equal to $\left\langle\beta, \alpha^{\vee}\right\rangle$. One can show, e.g. using the function $F_{\alpha}$ of Example 3.11, that $D_{\alpha}$ is moved exactly by the two simple roots $(\alpha, 0)$ and $(0,-\alpha)$ of $G \times G$, which are indeed orthogonal and add up to a spherical root.

Remark 5.9. We give another property of the combinatorics of $\Sigma(X)$. It follows from Propositions 2.11 and 3.2 that any spherical root $\sigma$ of a wonderful variety $X$ is the spherical root of a wonderful variety $Y$ of $\operatorname{rank} 1$, with the same associated parabolic subgroup $P$, i.e. $S^{p}(X)=S^{p}(Y)$.

As mentioned in Example 2.10, wonderful varieties of rank 1 are classified, and their spherical roots together with the sets $S^{p}$ are known. This imposes another condition on the possible elements of $\Sigma(X)$, and a compatibility condition between 
each single spherical root and the set $S^{p}(X)$. This condition will be given explicitly in Section 6.

The triple $\left(S^{p}(X), \Sigma(X), \Delta(X)\right)$, where often $\Delta(X)$ is replaced by the subset of colors moved by simple roots that are spherical roots, is called the spherical system of $X$. Luna suggested in [Lu01] a purely combinatorial notion of spherical systems, based on the properties stated in Proposition 5.6 and Remark 5.9.

In the same paper Luna also conjectured that spherical systems classify wonderful varieties, proving it for groups of type $A$. The conjecture has been proved in general in [Lo09] (uniqueness of a wonderful variety having a given spherical system) and [BP16] (existence of a wonderful variety having a given spherical system). We refer to [BP16] and references therein for details on this classification.

\section{LUNA DIAGRAMS}

As Luna observed in [Lu01], the above properties impose so stringent conditions on $S^{p}(X), \Sigma(X), \Delta(X)$, and the Cartan pairing, that it is possible to represent these objects entirely with diagrams "attached" to the Dynkin diagrams of $G$. These are now called Luna diagrams.

Let us explain how they are constructed. Colors are represented by circles, possibly filled; whether filled or not depends on $\Sigma(X)$ as explained below.

A circle corresponding to a color $D$ is drawn in the vicinity of a simple root $\alpha$ (which means below, above, or around $\alpha$ ) if $\alpha$ moves $D$. It can happen that more than one simple root, say $\alpha, \ldots, \alpha_{k}$, move $D$. In this case we represent $D$ as $k$ circles, each drawn in the vicinity of resp. $\alpha_{1}, \ldots, \alpha_{k}$, and joined by a line.

How a color $D$ is drawn in the vicinity of a simple root $\alpha$ that moves $D$ is decided in the following way, following Proposition 5.6.

(1) If $\alpha \in \Sigma(X)$, then $D$ is one of $D_{\alpha}^{+}, D_{\alpha}^{-}$. They are drawn resp. above and below $\alpha$.

(2) If $2 \alpha \in \Sigma(X)$, then $\alpha$ moves only $D$, which is drawn below $\alpha$.

(3) Otherwise $\alpha$ moves only $D$, which is drawn around $\alpha$.

Each spherical root $\sigma \in \Sigma(X)$ is a linear combination of simple roots with rational, non-negative coefficients (see [Lu01]), and if $G$ is adjoint then these linear combinations have integer coefficients, since spherical roots obviously belong to the root lattice in this case. The set of simple roots whose coefficient is positive is called the support of $\sigma$.

For simplicity we assume that $G$ is adjoint, and each spherical root $\sigma$ appearing in this case has its own symbol as in Table 1. This symbol is drawn on the part of the Dynkin diagram of $G$ corresponding to the support of $\sigma$. The presence of a given spherical root forces simple roots on its support to move some colors. These colors are then drawn in Table 1, and if in the table no color is explicitly drawn in the vicinity of a simple root $\alpha$, then $\alpha$ moves no color. This rule has actually two exceptions:

(1) in the sixth case, the spherical root $\alpha_{1}+\ldots+\alpha_{r}$ with support of type $\mathrm{B}_{r}$, the simple root $\alpha_{r}$ may move colors,

(2) in the ninth case, the spherical root $\alpha_{1}+2\left(\alpha_{2}+\ldots+\alpha_{r-1}\right)+\alpha_{r}$ with support of type $\mathrm{C}_{r}$, the simple root $\alpha_{1}$ may move colors.

In the table we mark these exceptions with an asterisk, which is however not reproduced in the actual Luna diagram. 
These remarks amount to the compatibility conditions between $\alpha \in S^{p}(X)$ and $\sigma \in \Sigma(X)$ mentioned in Remark 5.9, in the case where $\alpha$ is in the support of $\sigma$. If this is not the case, the only compatibility condition is the one given in Lemma 5.4.

Notice that some of the symbols of the table are realized simply by filling the circle corresponding to some color $D$ moved by a simple root $\alpha$ in the support of the spherical root. If a color does not constitute in this way the symbol of some spherical root, it is drawn not filled.

We also precise that a "zigzag" line appearing as the fourth case (the spherical root $\alpha_{1}+\ldots+\alpha_{r}$, where $\alpha_{1}, \ldots, \alpha_{r}$ form a subdiagram of type $\mathrm{A}_{r}$ ) is meant in our convention to join the two vertices $\alpha_{1}, \alpha_{r}$, not the two circles drawn around them. The two circles correspond to two different colors.

Much of the Cartan pairing is often deduced from Proposition 5.6, therefore only in specific situations we need to add some indications about it to the Luna diagram.

Following the proposition, the value of $c(D,-)$ for a color $D$ moved by a simple root $\alpha$ is entirely determined by $\alpha$, unless $\alpha \in \Sigma(X)$. Even in this case, where $\alpha$ moves the two colors $D_{\alpha}^{+}, D_{\alpha}^{-}$, it is enough to give the Cartan pairing for only one of them, since $c\left(D_{\alpha}^{ \pm},-\right)=\alpha^{\vee}-c\left(D_{\alpha}^{\mp},-\right)$. Finally, the value $c\left(D_{\alpha}^{ \pm}, \sigma\right)$ is positive for some $\sigma \in \Sigma(X)$ if and only if it is equal to 1 , the spherical root $\sigma$ is a simple root, and $D_{\alpha}^{ \pm}$also appears as $D_{\sigma}^{ \pm}$. Hence such values are deduced from the Luna diagram as we have described it so far.

It remains to include some indications on the value $c\left(D_{\alpha}^{ \pm}, \sigma\right)$ in the cases where it is not positive and $\left\langle\alpha^{\vee}, \sigma\right\rangle \neq 0$. In these cases we indicate the value $c\left(D_{\alpha}^{ \pm}, \sigma\right)=k$ by drawing $-k$ small arrows on the side of one of the circles representing the color, pointing at some simple root in the support of $\sigma$. We remark that negative values smaller than -1 are possible, as shown in Example 5.7.

A common practice in the literature is to draw these arrows only for the color $D_{\alpha}^{+}$ (the one drawn above $\alpha$ ), since this is enough to determine the value of the Cartan pairing for $D_{\alpha}^{-}$, as we have already observed. We will follow this convention here, but we point out that it is then slightly more cumbersome to recognize whether two different Luna diagrams (with equal $\Sigma$ and $\Delta$ ) give the same Cartan pairing or not. For this reason, indications for both colors are found in some references.

Example 6.1. For any spherical root of Table 1, one can find a description of the open $G$-orbit of the corresponding wonderful variety in [Wa96, Table 1]. Here we have already seen the cases $\alpha_{1}$ and $2 \alpha_{1}$ for $G=\mathrm{SL}(2)$ in Example 5.5. Let us see some more cases.

(1) The variety $X=\mathbb{P}\left(\mathbb{C}^{n+1}\right) \times \mathbb{P}\left(\left(\mathbb{C}^{n+1}\right)^{*}\right)$, under the diagonal action of $G=$ $\mathrm{SL}(n+1)$, is the wonderful completion of the homogeneous space $\mathrm{SL}(n+$ 1) $/ \mathrm{GL}(n)$ and has spherical root $\alpha_{1}+\ldots+\alpha_{n}$.

(2) The variety $X=\mathbb{P}(V)$, where $V$ is the irreducible 8-dimensional Spin(7)module, is wonderful under the action of $\mathrm{SO}(7)$, and has spherical root $\alpha_{1}+2 \alpha_{2}+3 \alpha_{3}$

(3) The variety $X=\mathbb{P}(U)$, where $U$ is the irreducible 7-dimensional $\mathrm{G}_{2}$-module, is wonderful under the action of $\mathrm{G}_{2}$, and has spherical root $4 \alpha_{1}+2 \alpha_{2}$.

Example 6.2. (1) Partial flag varieties $G / P$ with $P \supseteq B$ don't have spherical roots, and their colors are the Schubert divisors. The Luna diagram is therefore given only drawing a circle around each simple root that moves some Schubert divisor. For example, let $G$ be $\mathrm{SL}(4)$ with simple roots 
TABLE 1. Diagrams of spherical roots

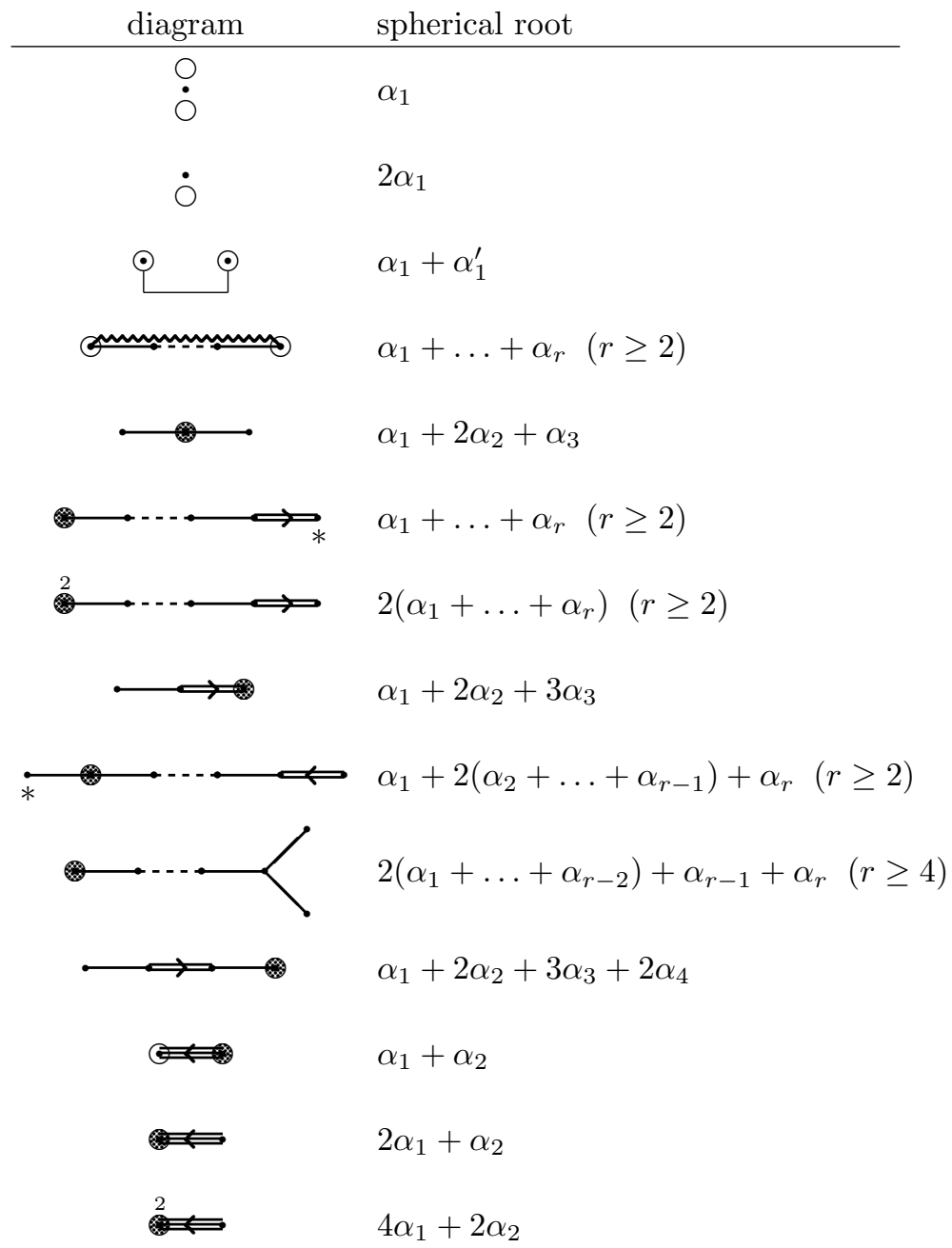

$\alpha_{1}, \alpha_{2}, \alpha_{3}$. The Luna diagram of $X=G / B$ is

$$
\odot \odot
$$

Let $P$ be the parabolic subgroup of SL(4) containing $B$ and with Levi subgroup having only the simple root $\alpha_{3}$. Then the Luna diagram of $X^{\prime}=$ $G / P$ is

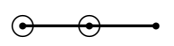

(2) The diagram

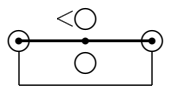


represents the set of spherical roots $\left\{\alpha_{1}+\alpha_{3}, \alpha_{2}\right\}$, and the set of colors $\left\{D, D_{\alpha_{2}}^{+}, D_{\alpha_{2}}^{-}\right\}$with Cartan pairing

\begin{tabular}{c|cc} 
& $\alpha_{1}+\alpha_{3}$ & $\alpha_{2}$ \\
\hline$D$ & 2 & -1 \\
$D_{\alpha_{2}}^{+}$ & -1 & 1 \\
$D_{\alpha_{2}}^{-}$ & -1 & 1
\end{tabular}

(3) The diagram

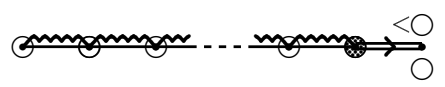

(for $G$ of type $\mathrm{B}_{n}$ ) represents the set of spherical roots $\left\{\alpha_{1}+\alpha_{2}, \alpha_{2}+\right.$ $\left.\alpha_{3}, \ldots, \alpha_{n-2}+\alpha_{n-1}, \alpha_{n-1}+\alpha_{n}, \alpha_{n}\right\}$. Any color takes the values of some simple coroot, except for $D_{\alpha_{n}}^{+}, D_{\alpha_{n}}^{-}$, which take the following values:

\begin{tabular}{c|cccccc} 
& $\alpha_{1}+\alpha_{2}$ & $\ldots$ & $\alpha_{n-3}+\alpha_{n-2}$ & $\alpha_{n-2}+\alpha_{n-1}$ & $\alpha_{n-1}+\alpha_{n}$ & $\alpha_{n}$ \\
\hline$D_{\alpha_{n}}^{+}$ & 0 & $\ldots$ & 0 & -1 & 0 & 1 \\
$D_{\alpha_{n}}^{\alpha_{n}}$ & 0 & $\ldots$ & 0 & -1 & 0 & 1
\end{tabular}

Notice that $c\left(D_{\alpha_{n}}^{ \pm}, \alpha_{n-1}+\alpha_{n}\right)=0$ because if one of these values were non-zero, one would be strictly positive (since $\left\langle\alpha_{n}^{\vee}, \alpha_{n-1}+\alpha_{n}\right\rangle=0$ ), contradicting Proposition 5.6.

(4) The diagram

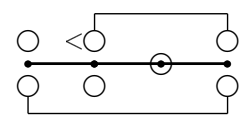

for $G=\mathrm{SL}(5)$ with simple roots $\alpha_{1}, \ldots, \alpha_{4}$ represents $\Sigma(X)=\left\{\alpha_{1}, \alpha_{2}, \alpha_{4}\right\}$ and $\Delta(X)=\left\{D_{\alpha_{1}}^{+}, D_{\alpha_{1}}^{-}=D_{\alpha_{4}}^{-}, D_{\alpha_{2}}^{+}=D_{\alpha_{4}}^{+}, D_{\alpha_{2}}^{-}\right\}$with Cartan pairing

\begin{tabular}{c|ccc} 
& $\alpha_{1}$ & $\alpha_{2}$ & $\alpha_{4}$ \\
\hline$D_{\alpha_{1}}^{+}$ & 1 & 0 & -1 \\
$D_{\alpha_{1}}^{-}$ & 1 & -1 & 1 \\
$D_{\alpha_{2}}^{+}$ & -1 & 1 & 1 \\
$D_{\alpha_{2}}^{-}$ & 0 & 1 & -1
\end{tabular}

\section{Morphisms}

In [Kn91] dominant morphisms with connected fibers between spherical varieties are studied. The special case of morphisms between wonderful varieties was derived from loc.cit. in [Lu01]. We report this case in this section.

Let $X$ be a wonderful variety. Recall that we have defined the convex cone $V$ generated inside $N=\operatorname{Hom}_{\mathbb{Z}}(\Xi(X), \mathbb{Z})$ by the elements $-\sigma_{1}^{*}, \ldots,-\sigma_{r}^{*}$, where $\sigma_{1}^{*}, \ldots, \sigma_{r}^{*}$ is the dual basis of $\Sigma(X)$.

Definition 7.1. Let $X$ be a wonderful variety with open $G$-orbit $G / H$. If $K$ is a subgroup of $G$ containing $H$, we denote by $\Delta_{K}$ the set of colors of $G / H$ mapped dominantly to $G / K$ by the natural map $G / H \rightarrow G / K$, and $\mathcal{C}_{K}$ the subset of $N$ consisting of all elements vanishing on $B$-eigenvalues of $B$-eigenvectors in $\mathbb{C}(G / K)$.

Definition 7.2. Let $\mathcal{C}$ be a vector subspace of $N$, and $\Delta^{\prime} \subseteq \Delta(X)$. The couple $\left(\mathcal{C}, \Delta^{\prime}\right.$ ) is a colored subspace (of $N$ ) if $C$ is generated, as a convex cone, by finitely many elements of $V$ together with the elements $c(D,-)$ for $D \in \Delta^{\prime}$. 
Theorem 7.3 ([Kn91]). Let $X$ be a wonderful variety with open $G$-orbit $G / H$. The map $K \mapsto\left(\mathcal{C}_{K}, \Delta_{K}\right)$ is a bijection between the set of subgroups $K$ of $G$ containing $H$ such that $K / H$ is connected, and the set of colored subspaces of $N$. Moreover, $K$ has finite index in $N_{G}(K)$ if and only if the image of $V$ in $N / \mathcal{C}_{K}$ is strictly convex.

Definition 7.4. A subset $\Delta^{\prime} \subseteq \Delta(X)$ is distinguished if $-V$ intersects the relative interior $E_{0}$ of the cone $E$ generated by the elements $c(D,-)$ for $D \in \Delta^{\prime}$.

Lemma 7.5. Let $F_{1}$ and $F_{2}$ be polyhedral convex cones in a finite-dimensional rational vector space. The convex cone generated by $F_{1}$ and $F_{2}$ is a vector subspace if and only if the relative interiors of $-F_{1}$ and of $F_{2}$ intersect.

Proof. If the relative interior of $-F_{1}$ and of $F_{2}$ intersect, then the convex cone $R$ generated by $F_{1}$ and $F_{2}$ contains the two vector subspaces generated resp. by $F_{1}$ and $F_{2}$. It follows that $R$ is the vector subspace generated by $F_{1}$ together with $F_{2}$.

Vice versa, suppose that $R$ is a vector subspace, and consider a point $x$ in the relative interior of $-F_{1}$. Then $x \in R$, so it is a sum $x=f_{1}+f_{2}$ where $f_{i} \in F_{i}$ for all $i \in\{1,2\}$. The point $f_{2}=x-f_{1}$ is in the relative interior of $-F_{1}$, and also in $F_{2}$.

By symmetry, we also have that $-F_{1}$ intersects the relative interior of $F_{2}$, let $z$ be a point in this intersection. If $z=f_{2}$ then we are done, otherwise any point on the segment joining $f_{2}$ and $z$ (possibly except for the endpoints) lyes in the relative interiors of $-F_{1}$ and of $F_{2}$.

Proposition 7.6 ([Lu01]). Let $X$ be a wonderful variety with open $G$-orbit $G / H$, and $\Delta^{\prime} \subseteq \Delta(X)$. Then $\Delta^{\prime}$ is distinguished if and only if there exists a unique vector subspace $\mathcal{C}$ of $N$ such that $\left(\mathcal{C}, \Delta^{\prime}\right)$ is a colored subspace, and the image of $V$ in $N / \mathcal{C}_{K}$ is strictly convex.

Proof. First we notice that, if $\mathcal{C}$ is a vector subspace of $N$, the image of $V$ in $N / \mathcal{C}$ is strictly convex if and only if $\mathcal{C} \cap(-V)$ is a face of $-V$.

Now assume that there exists a colored subspace $\left(\mathcal{C}, \Delta^{\prime}\right)$ of $N$ such that $\mathcal{C} \cap(-V)$ is a face $F$ of $-V$. Denote as above by $E$ the cone generated by the elements $c(D,-)$ for $D \in \Delta^{\prime}$, and $E_{0}$ the relative interior of $E$. Then $F$ generates $\mathcal{C}$ as a convex cone together with $E$, which implies that $-F$ intersects $E_{0}$ by Lemma 7.5. Hence $\Delta^{\prime}$ is distinguished.

Vice versa, suppose that $\Delta^{\prime}$ is distinguished, so $-E_{0}$ intersects $V$. Suppose that there exist two faces $F_{1}, F_{2}$ of $V$ such that their relative interiors intersect $-E_{0}$. Then the relative interior of the face of $V$ generated by $F_{1}$ and $F_{2}$ also intersects $-E_{0}$.

It follows that there exists a unique maximal face $F$ of $V$ such that its relative interior intersects $-E_{0}$. Then $F$ and $E$ generate a vector subspace $\mathcal{C}$ of $N$ by Lemma 7.5.

Finally, we prove the uniqueness of $\mathcal{C}$. Let $\left(\mathcal{C}^{\prime}, \Delta^{\prime}\right)$ be a colored subspace such that $\mathcal{C}^{\prime} \cap V$ is a face $F^{\prime}$ of $V$. Since $\mathcal{C}^{\prime}$ contains $-E_{0}$, we have that $F^{\prime}$ contains $-E_{0} \cap V$, which intersects the relative interior of $F$. It follows that $F^{\prime}$ contains $F$. On the other hand, $F^{\prime}$ and $E$ generate $\mathcal{C}^{\prime}$ as a convex cone, which implies that the relative interior of $F^{\prime}$ intersects $-E_{0}$. By maximality of $F$, we have $F=F^{\prime}$ and $\mathcal{C}=\mathcal{C}^{\prime}$.

Example 7.7. The set of all colors $\Delta(X)$ is always distinguished. One can prove this fact by noticing that $\Delta(X)$ is the subset of colors $\Delta_{G}$ corresponding to the 
inclusion $H \subseteq G$. A combinatorial proof, due to P. Bravi, goes as follows. Thanks to Proposition 5.6, the convex cone $E$ corresponding to $\Delta(X)$ contains the restrictions $\left.\alpha^{\vee}\right|_{\Xi(X)}$ for all $\alpha \in S \backslash S^{p}(X)$. Therefore there exists an element $x$ of $E$ that is strictly positive on all simple roots not in $S^{p}(X)$. As one can check in Table 1, this implies that $x$ is strictly positive on all elements of $\Sigma(X)$.

As discussed in details in [Lu01], some subgroups $K \supseteq H$ (such that $K / H$ is connected) are such that $G / K$ admit a wonderful completion $Y$. A necessary condition recalled in Theorem 4.3 is that $K$ has finite index in its normalizer, and a remarkable theorem by Bravi (see [B13]) implies that this condition is also sufficient.

We end this section reporting on this result, and on the determination of the spherical roots of $Y$ done by Luna in [Lu01].

Definition 7.8. A distinguished subset of colors $\Delta^{\prime} \subseteq \Delta(X)$ is good if the monoid

$$
\left\{\sigma \in \operatorname{span}_{\mathbb{N}} \Sigma(X) \mid c(D, \sigma)=0 \text { for all } D \in \Delta^{\prime}\right\}
$$

is free. If this is the case, we denote by $\Sigma(X) / \Delta^{\prime}$ the basis of this monoid.

Proposition 7.9 ([Lu01]). Let $\Delta^{\prime} \subseteq \Delta(X)$ be a good distinguished subset of colors, and $K \supseteq H$ the corresponding subgroup of $G$. Then $G / K$ admits a wonderful completion $Y$. Moreover, the natural morphism $G / H \rightarrow G / K$ extends to a surjective equivariant map $X \rightarrow Y$ with connected fibers, and we have

$$
\Sigma(Y)=\Sigma(X) / \Delta^{\prime}
$$

The set of colors of $Y$ in the above proposition is also denoted by $\Delta(X) / \Delta^{\prime}$.

Theorem 7.10 ([B13]). All distinguished subsets of colors are good.

We may collect the above results in the following.

Corollary 7.11. Let $X$ be a wonderful G-variety. The map defined in Theorem 7.3 induces a bijection between the set of surjective, equivariant morphisms with connected fibers from $X$ to another wonderful variety and the set of distinguished subsets of $\Delta(X)$.

Example 7.12. (1) The Luna diagram

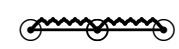

for $G=\mathrm{SL}(4)$ with simple roots $\alpha_{1}, \alpha_{2}, \alpha_{3}$, represents the spherical roots $\Sigma(X)=\left\{\alpha_{1}+\alpha_{2}, \alpha_{2}+\alpha_{3}\right\}$ and the colors $\Delta(X)=\left\{D_{1}, D_{2}, D_{3}\right\}$ with $D_{i}=\left.\alpha_{i}^{\vee}\right|_{\Xi(X)}$ for all $i \in\{1,2,3\}$. The Cartan pairing is therefore

\begin{tabular}{c|cc} 
& $\alpha_{1}+\alpha_{2}$ & $\alpha_{2}+\alpha_{3}$ \\
\hline$D_{1}$ & 1 & -1 \\
$D_{2}$ & 1 & 1 \\
$D_{3}$ & -1 & 1
\end{tabular}

Let us choose $\mathrm{Sp}(4) \subset \mathrm{SL}(4)$ in such a way that $\mathrm{Sp}(4) \cap B$ is a Borel subgroup of $\operatorname{Sp}(4)$. Then the above Luna diagram corresponds to the wonderful completion $X$ of the homogeneous space $\mathrm{SL}(4) / H$, where $H$ is the parabolic subgroup of $\mathrm{Sp}(4) \cdot Z(\mathrm{SL}(4))$ containing $\operatorname{Sp}(4) \cap B$ and corresponding to the long simple root. The subset $\Delta^{\prime}=\left\{D_{1}, D_{3}\right\}$ is distinguished, and the monoid $\left\{\sigma \in \operatorname{span}_{\mathbb{N}} \Sigma(X) \mid c(D, \sigma)=0\right.$ for all $\left.D \in \Delta^{\prime}\right\}$ is free, with basis 
the sum $\alpha_{1}+2 \alpha_{2}+\alpha_{3}$ of the two spherical roots of $X$. The corresponding surjective equivariant morphism $X \rightarrow Y$ corresponds to the inclusion $H \subset$ $K=\mathrm{Sp}(4) \cdot Z(\mathrm{SL}(4))$, and $Y$ has Luna diagram

(2) The Luna diagram

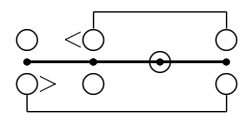

corresponds to the subgroup $H \subseteq \mathrm{SL}(5)$ of the matrices of the form

$$
\left(\begin{array}{ccc}
\lambda_{1} A & 0 & * \\
0 & \lambda_{2} & * \\
0 & 0 & \lambda_{3} A
\end{array}\right)
$$

where $A \in \mathrm{SL}(2)$ and $\lambda_{1}, \lambda_{2}, \lambda_{3} \in \mathbb{C}^{*}$ satisfy $\lambda_{1}^{2} \lambda_{2} \lambda_{3}^{2}=1$. The subset of colors $\Delta_{1}=\left\{D_{\alpha_{1}}^{-}, D_{\alpha_{2}}^{+}\right\}$is distinguished. The quotients $\Sigma(X) / \Delta_{1}$ and $\Delta(X) / \Delta_{1}$ are represented by the Luna diagram

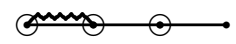

and correspond to $K_{1} \supset H$ of the matrices of the form

$$
\left(\begin{array}{ccc}
\lambda_{1} A & 0 & * \\
0 & \lambda_{2} & * \\
0 & 0 & \lambda_{3} B
\end{array}\right)
$$

where $B \in \mathrm{SL}(2)$. Also the subset of colors $\Delta_{2}=\left\{D_{\alpha_{1}}^{+}, D_{\alpha_{2}}^{+}\right\}$is distinguished, and gives

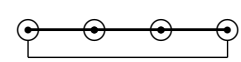

which corresponds to $K_{2} \supset H$ of the matrices of the form

$$
\left(\begin{array}{ccc}
\lambda_{1} A & * & * \\
0 & \lambda_{2} & * \\
0 & 0 & \lambda_{3} A
\end{array}\right)
$$

\section{Minimal MORPHismS}

Let $X$ be a wonderful $G$-variety with open $G$-orbit $G / H$. Minimal non-empty distinguished subsets of $\Delta(X)$ correspond to subgroups $K \subseteq G$ such that $K \supsetneq H$ and $K / H$ is connected, $G / K$ has a wonderful completion $Y$, and $K$ is minimal with respect to these properties. We may assume that it has a Levi subgroup $L_{K}$ containing a Levi subgroup $L_{H}$ of $H$.

Such subgroups $K$ have been used crucially in the classification of wonderful varieties. Indeed, they are involved in one of the key technical steps both of the uniqueness part of the classification (see [Lo09]), and of the existence part (see [BP14, Section 5.3]). In both parts, loosely speaking, the approach is to use induction (e.g. on the dimension of $G / H$ ), showing existence and uniqueness (up to 
conjugation) of a subgroup $H$ corresponding to some data $S^{p}(X), \Sigma(X), \Delta(X)$, assuming them for $K$ and the data $S^{p}(Y), \Sigma(Y), \Delta(Y)$.

This approach is effective under an additional fundamental assumption: that $K$ and $L$ have the same Levi subgroup, possibly up to some $\mathbb{C}^{*}$-factors, and that $K^{u} \supsetneq H^{u}$ (see case $(\mathscr{L})$ of Proposition 8.1 for a precise statement).

This suggests the importance of distinguishing between different types of inclusions $K \supsetneq H$, according to the behavior of Levi subgroups and of the unipotent radicals of $K$ and $H$, and motivates Proposition 8.1 below.

Proposition 8.1 ([BL11]). Under the above assumptions, the groups $H$ and $K$ satisfy one of the following statements.

(P) We have $H^{u} \supsetneq K^{u}$. In this case $H$ is a maximal proper parabolic subgroup of $K$, and $Z\left(L_{H}\right)^{\circ} \supsetneq Z\left(L_{K}\right)^{\circ}$.

$(\mathscr{R})$ We have $H^{u}=K^{u}$. In this case $H^{r}=K^{r}$, and $H / H^{r}$ is very reductive in $K / K^{r}$.

(L) We have $H^{u} \subsetneq K^{u}$. In this case $L_{K}=L_{H} Z(H)^{\circ}$ and $Z\left(L_{H}\right)^{\circ} \subseteq Z\left(L_{K}\right)^{\circ}$, and $K^{u} / H^{u}$ is $L_{H}$-equivariantly isomorphic to a simple $L_{H}$-module.

In the above proposition, we also say that the inclusion $H \subset K$ is of type $(\mathscr{P})$, $(\mathscr{R})$, or $(\mathscr{L})$, according to which of the statements holds.

The following corollary follows immediately. We recall that the defect $d(X)$ of a wonderful variety $X$ is defined as $d(X)=|\Delta(X)|-|\Sigma(X)|$.

Corollary 8.2. Denote by $Y$ the wonderful completion of $G / K$. Then the following hold.

(1) If $d(X)<d(Y)$ then the inclusion $H \subset K$ is of type (L).

(2) If the inclusion is of type $(\mathscr{R})$ then $d(X)=d(Y)$.

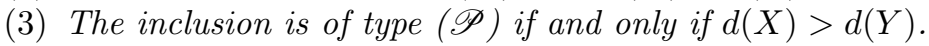

Example 8.3. (1) The subset $\Delta^{\prime}$ of Example 7.12(1) gives an inclusion $H \subset K$ of type $(\mathscr{P})$, and the subset $\Delta_{2}$ of Example $7.12(2)$ gives an inclusion $H \subset K_{2}$ of type $(\mathscr{R})$.

(2) The subset $\Delta_{1}$ of Example 7.12(2) gives an inclusion $H \subset K_{1}$ of type $(\mathscr{L})$. Notice that here $Z\left(L_{H}\right)^{\circ}=Z\left(L_{K_{1}}\right)^{\circ}$, showing that parts (1) and (2) of Corollary 8.2 are not equivalences in general.

(3) An example where $Z\left(L_{H}\right)^{\circ} \subsetneq Z\left(L_{K}\right)^{\circ}$ is the one discussed in Example 5.7. Let us discuss the case $G=\mathrm{SL}(4)$, which has Luna diagram

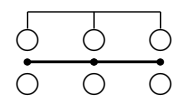

We can describe $H$ as the subgroup of matrices of the form

$$
\left(\begin{array}{cccc}
\lambda_{1} & a_{1} & * & * \\
0 & \lambda_{2} & a_{2} & * \\
0 & 0 & \lambda_{3} & a_{3} \\
0 & 0 & 0 & \lambda_{4}
\end{array}\right)
$$

where $a_{1}, a_{2}, a_{3} \in \mathbb{C}$ satisfy $a_{1}+a_{2}+a_{3}=0$, and $\lambda_{1}, \ldots, \lambda_{4} \in \mathbb{C}^{*}$ satisfy $\lambda_{1} \cdots \lambda_{4}=1$ and also $\lambda_{1} / \lambda_{2}=\lambda_{2} / \lambda_{3}=\lambda_{3} / \lambda_{4}$. Notice that one can also take another linear combination of $a_{1}, a_{2}, a_{3}$ (with coefficients all non-zero) instead of $a_{1}+a_{2}+a_{3}$, and obtain a subgroup of SL(4) conjugated to $H$. 
The subset of colors $\Delta^{\prime}=\left\{D_{1}^{+}=D_{2}^{+}=D_{3}^{+}\right\}$is distinguished, and the corresponding subgroup is $K=B$. Here the inclusion $H \subset K$ is of type $(\mathscr{L})$ and we have $Z\left(L_{H}\right)^{\circ} \subsetneq Z\left(L_{K}\right)^{\circ}$. In this situation observe that $Z\left(L_{K}\right)^{\circ}$ does not normalize $H$, in accordance with Theorem 4.3. More precisely, the unipotent radical $H^{u}$ is not stable under conjugation by $Z\left(L_{K}\right)^{\circ}$, because it contains a submodule of $K^{u}$ that is "diagonal" in a sum of $L_{K^{-}}$ submodules contained in $K^{u}$, all isomorphic as $\left(L_{K}^{\circ}, L_{K}^{\circ}\right)$-modules but not as $L_{K}$-modules.

\section{REFERENCES}

[Ah83] D.N. Ahiezer, Equivariant completions of homogeneous algebraic varieties by homogeneous divisors, Ann. Global Anal. Geom. 1 (1983), no. 1, 49-78.

[Av15] R. Avdeev, Strongly solvable spherical subgroups and their combinatorial invariants, Selecta Math. (N.S.) 21, no. 3, (2015), 931-993.

[B13] P. Bravi, Primitive spherical systems, Trans. Amer. Math. Soc. 365 (2013), 361-407.

[BL11] P. Bravi, D. Luna, An introduction to wonderful varieties with many examples of type F4, J. Algebra 329 (2011) 4-51.

[BP14] P. Bravi, G. Pezzini, Wonderful subgroups of reductive groups and spherical systems, J. of Algebra 409 (2014), 101-147.

[BP16] P. Bravi, G. Pezzini, Primitive wonderful varieties, Math. Z. 282 (2016), no. 3-4, 10671096.

[Br88] M. Brion, On spherical varieties of rank one (after D. Ahiezer, A. Huckleberry, D. Snow), Group actions and invariant theory (Montreal, PQ, 1988), CMS Conf. Proc., 10, Amer. Math. Soc., Providence, RI, 1989, 31-41.

[Br89] M. Brion, Groupe de Picard et nombres caractéristiques des variétés sphériques. Duke Math. J. 58 (1989), no. 2, 397-424.

[Br90] M. Brion, Vers une généralisation des espaces symétriques, J. Algebra 134 (1990), no. 1, $115-143$.

[Br07] M. Brion, Log homogeneous varieties, Actas del XVI Coloquio Latinoamericano de Álgebra, 1-39, Revista Matemática Iberoamericana, Madrid, 2007.

[BLV86] M. Brion, D. Luna, Th. Vust, Espaces homogènes sphériques, Invent. Math. 84 (1986), $617-632$.

[BrPa87] M. Brion, F. Pauer, Valuations des espaces homogènes sphériques, Comment. Math. Helvetici 62 (1987), 265-285.

[DP83] C. De Concini, C. Procesi, Complete symmetric varieties, Invariant theory (Montecatini, 1982), Lecture Notes in Math., 996, Springer, Berlin, 1983, 1-44.

[Kn91] F. Knop., The Luna-Vust theory of spherical embeddings, Proceedings of the Hyderabad Conference on Algebraic Groups (Hyderabad, 1989), 225-249, Manoj Prakashan, Madras, 1991.

[Kn96] F. Knop, Automorphisms, root systems, and compactifications of homogeneous varieties, J. Amer. Math. Soc. 9 (1996), no. 1, 153-174.

[Kn14] F. Knop, Spherical roots of spherical varieties, Ann. Inst. Fourier (Grenoble) 64 (2014), $2503-2526$.

[KKV89] F. Knop, H. Kraft, Th. Vust, The Picard group of a G-variety, Algebraische Transformationsgruppen und Invariantentheorie (H. Kraft, P. Slodowy, T. A. Springer, eds.), DMV Seminar, vol. 13, pp. 77-88, Birkhäuser, Basel-Boston-Berlin, 1989.

[Lo09] I. Losev, Uniqueness property for spherical homogeneous spaces, Duke Mathematical Journal 147 (2009), no. 2, 315-343.

[Lo09b] I. V. Losev, Demazure embeddings are smooth, Internat. Math. Res. Notices (2009), no. $14,2588-2596$.

[Lu73] D. Luna, Slices étales, Mémoires de la S.M.F., 33 (1973), 81-105.

[Lu96] D. Luna, Toute variété magnifique est sphérique, Transform. Groups 1 (1996), no. 3, 249258.

[Lu97] D. Luna, Grosses cellules pour les variétés sphériques, Algebraic groups and Lie groups, Austral. Math. Soc. Lect. Ser., 9, Cambridge Univ. Press, Cambridge, 1997, 267-280. 
[Lu01] D. Luna, Variétés sphériques de type A, Inst. Hautes Études Sci. Publ. Math. 94 (2001), $161-226$.

[MJ88] L. Moser-Jauslin, Some almost homogeneous group actions on smooth complete rational surfaces, L'Enseignement Mathématique 34 (1988), 313-332.

[Pe09] G. Pezzini, Automorphisms of wonderful varieties, Transform. Groups 14 (2009), no. 3, $677-694$.

[Se51] J. G. Semple, The variety whose points represent complete collineations of $S_{r}$ on $S_{r}^{\prime}$, Univ. Roma, Ist. Naz. Alta Mat. Rend. Mat. e Appl. (5) 10 (1951), 201-208.

[Su74] H. Sumihiro, Equivariant completion, J. Math. Kyoto Univ. 14 (1974), 1-28.

[Wa96] B. Wasserman, Wonderful varieties of rank two, Transform. Groups 1 (1996), no. 4, 375403. 
To the referee

I thank the referee for the careful reading and many remarks. All comments of the report have been taken into account, here are some details:

(1) I have added some examples where requested.

(2) I have now drawn Luna diagrams always following a common practice, which is to omit the small "arrows" on the side of colors drawn below a simple root (I was not consistent on this in the first version). A comment on this has been added to the description of Luna diagrams before Example 6.1.

(3) I have corrected small errors in Remark 3.10 and Example 3.11.

(4) I have expanded the last statement of the first version of the paper, and made it into a couple of paragraphs on the proof of the classification, at the beginning of Section 8.

Dipartimento di Matematica "G. Castelnuovo", Università di Roma "La Sapienza", Piazzale Aldo Moro 5, 00185 Roma, Italy

E-mail address: pezzini@mat.uniroma1.it 